\title{
Hermann Pfütze
}

\section{Zur soziologischen Produktion des Nichtwissens* - von der Gesellschaftstheorie zum Katastrophenwissen -}

Zusammenfassung: Zum Glück leidet auch die Soziologie an dem Bewußtsein, schwächer zu sein als ihr Gegenstand. Die Unerforschlichkeit der Gesellschaft, ihre weder plan-noch steuerbaren Wege und Umwege der Vergesellschaftung, zwingen die Soziologie zu stetiger Selbstreflexion.Je mehr Wissen indes über die Gefahren und Zerstörungen der globalen Risikogesellschaft produziert wird, desto schwieriger wird nicht nur eine Theorie davon, sondern um so größer auch der Wunsch, wissensmäßig stärker zu sein als der Gegenstand - sozusagen für alle Fälle alles im Datenspeicher zu haben. Je mehr Wissen die Soziologie produziert, desto weniger weiß sie freilich die Rettung: Wie die Gesellschaft sich nicht zerstört, ist nicht zu sagen.D.h., die Gesellschaft selbst ist theoretisch und nicht nur Gegenstand von Theorien. Das für die Soziologie konstitutive Verhältnis zwischen Wissensproduktion und der Fähigkeit, Gewißheiten fahren zu lassen, wird im folgenden ein wenig erörtert.

1.

Der >Paradigmenwechsel $<$ in den soziologischen Modernisierungstheorien z.B. von der klassentheoretisch fundierten Kapitalismuskritik der 60er Jahre zur gefahrentheoretisch fundierten Handlungstheorie der 80er ist, wissenssoziologisch gesehen, nicht nur ein Umsatteln vom Gedenken dessen, was passiert ist, auf den Gedanken an das, was nicht passieren darf. Sondern er ist auch ein Stück weit Verlust der Kritik zugunsten der Affirmation. Nicht der Kapitalismus hat abgewirtschaftet, sondern die Hoffnung, ihn zu widerlegen. Mithin ist es weniger nötig zu erklären, warum der Kapitalismus weltweit erfolgreich ist, als vielmehr zu wissen, womit man rechnen muß. Die Rede von »Gefahrenlagen« statt von Klassenlagen kann freilich nicht davon absehen, daß das Leben der ärmeren Klassen nach wie vor größeren Gefahren ausgesetzt ist, weil ihr Gefährdungswissen geringer ist und die Möglichkeiten, ungefährlicher zu leben, für sie zu teuer sind.

Auch die Soziologie leidet an der »Gebrechlichkeit der sogenannten Humanwissenschaften«, die Roland Barthes so erklärt:

》... es sind Wissenschaften vom Nichtvorhersehbaren (daher die Enttäuschungen und das taxonomische Unbehagen der Ökonomie) - wodurch die Vorstellung von Wissenschaft unmittelbar beeinträchtigt wird; selbst die Wissenschaft von den Begierden, die Psychoanalyse, wird unweigerlich eines Tages sterben, ... denn die Begierde ist stärker als ihre Interpretation«. (Barthes 1980, S. 43)

Das heißt nicht, daß es eines Tages keine Wissenschaft mehr geben werde, die sich mit den Begierden - oder mit dem Kapitalismus - beschäftigt; es muß nur nicht immer 
die Psychoanalyse - oder die Ökonomie - sein. Wer weiß, ob die sog. Humanwissenschaften nicht wieder abgelöst werden von einer neuen, inhumanen Trias der Körper-, Glaubens- und Gesetzeswissenschaften? Das darf zwar nicht passieren, aber man kann es nicht wissen. Der Impuls, dies zu verhindern, artikuliert sich vielleicht in sog. Handlungstheorien, aber denen mangelt es aus diesem teleologischen Grund an Theorie (vgl. Schnädelbach 1989, S. 20 f.).

Die Gebrechlichkeit der Soziologie liegt an den prinzipiell nicht vorhesehbaren, weder plan- noch steuerbaren Wegen und Umwegen der Vergesellschaftung. Die Gesellschaft in der DDR z.B. löst sich gegenwärtig nicht auf, sondern sie $>$ weiß< noch nicht, wie sie sich neu vergesellschaftet, während sie es doch tut. Diese Unerforschlichkeit der Gesellschaft zwingt die Soziologie zu stetiger Selbstreflexion. Je mehr Wissen und Wissenserwerbstechnik die Sozialwissenschaften produzieren, desto schwieriger wird ullenbar die Theoriebildung. Der Wunsch z.B. Luhmanns, mit möglichst wenig Empirie auszukommen, ist nicht nur verständlich, sondern auch theoriefreundlich. Zwar gibt es Gesetzmäßigkeiten, auf die Theorie sich stützen kann, wie z.B. die kapitalistische Mehrwertproduktion oder die sozialen und psychischen Anpassungsund Abwehrmechanismen. Aber sie funktionieren eben nicht, wie die Newtonsche Mechanik, immer gleich, sondern von Fall zu Fall und von Tag zu Tag verschieden. Das Kapital modernisiert zur Zeit seine Akkumulationsweisen drastisch und global; und die Gefühle im Umgang mit ökologischen Gefahren und mit nichtkapitalistischen Gesellschaftsversuchen waren vor zwanzig Jahren anders zusammengesetzt als heute.

Auch die Soziologie lebt mithin von dem Bewußtsein, schwächer zu sein als ihr Gegenstand. Sie kann auch gar nicht stärker sein - das wäre der schreckliche Fall vollendeter Reduktion von Komplexität, der systematischen Auflösung des Sozialen in Letztelemente, software bits.

Nun neigen alle Wissenschaften dazu, das ist ihr Trieb, bis zum Kern oder auf den Grund ihrer Gegenstände erkennend vorzustoßen. In den Sprach- und Sozialwissenschaften ist das zwar nicht so gefährlich wie in der Atomphysik und Biochemie; die Suche nach vermeintlichen Elementarteilchen führt aber auch hier zu Endstationen. Nicht nur in der Atom- und Zellkernforschung rührt die Erkenntnis des Ursprungs der Materie und des Lebens zugleich an ihr Ende. Auch z.B. in der Semiotik und Informatik ist Sprachlosigkeit das Ergebnis der Elementarisierung und Digitalisierung der Sprache. Und in der Soziologie führt z.B. Luhmanns Versuch, Handeln und Denken in kleinstmögliche Letztelemente zu zerlegen, damit in jedem Augenblick die Wahl auch eines anderen Anschlusses möglich sei, zur zwangsneurotischen Denkfigur des »stehenden Pfeils« (Fachinelli 1981): Jederzeit alles anhalten, zerlegen, rückgängig und ungeschehen machen zu können. Das Paradox des Zeno, daß der Pfeil nicht fliege, sondern in jedem Augenblick an einem fixen Punkt >stehe , formuliert die Grundangst aller Wissenschaft, nämlich unterzugehen im Meer der Ungewißheiten ihrer Gegenstände. Darum muß der Pfeil stehen. Sein Flug wird umdefiniert in eine unendliche Serie von Gewißheitstests. (Vgl. Luhmann 1984, S. 117; und Pfütze, 1987) In allem Unheil, das ihre Gegenstände anrichten, ist es das Glück der Humanwissen- 
schaften, daß sie schwächer sind: daß z.B. die Begierden stärker sind als die Psychoanalyse, daß der Verwertungstrieb des Kapitals als bloße Mehrwertrate sich nicht fassen läßt, daß soziales Leben nicht in seinen empirischen Daten aufgeht. Je mehr die Soziologie Wissen sammelt und produziert über die selbstzerstörerische Dynamik moderner Industriegesellschaften, je genauer die sog. Risikogesellschaft absehbar wird, desto weniger wei $\beta$ sie die Rettung. Wie Gesellschaft sich nicht zerstört, ist nicht vorhersehbar. Gerade die Zerstörungsprognosen zeichnen sich dadurch aus, daß sie so wahrscheinlich sind wie ihr Gegenteil.

Die Gebrechlichkeit der Soziologie, ihre Beeinträchtigung durch die unberechenbare Stärke der Gesellschaft, ist freilich auch Antrieb ihrer >Paradigmenwechsel .

Das Verhältnis zwischen dem Tempo der Paradigmenwechsel und Umfang und Qualität der Wissensproduktion folgt dabei einer sog. logistischen Kurve. D.J. de Solla Prices Untersuchungen des Wissenswachstums in den Naturwissenschaften während des 19. und 20. Jahrhunderts verdeutlichen, mutatis mutandis, auch das gegenwärtige Problem der Soziologie: soviel wie nie zuvor zu wissen über Gesellschaften, ohne ihnen theoretisch gewachsen zu sein. Sehr verkürzt gesagt, sieht der steile Teil der logistischen Kurve etwa so aus: In Zeiten exponentiellen Wachstums, etwa während eines Paradigmenwechsels, der von der ganzen Zunft mit Emphase und viel Produktion von Literatur und Forschungsmaterial mitgemacht wird, sind die Halbwertzeiten und Verfallsdaten des größten Teils dieses Wissens relativ kurz-gemessen daran, wie häufig in welcher Zeit diese Literatur zitiert, diese Ergebnisse weiterverwendet werden. Die Kurve flacht daraufhin ab, sie nähert sich ikrer Sättigungsgrenze. - D.h. diese Daten und Theorieversuche, z.B. heute über Katastrophenwissen und Handeln, haben zwar etwas gebracht, aber sie sind nicht generativ. Auch mit gesteigertem Forschungsaufwand und gegenseitigem Zitieren ist nicht viel Neues mehr über die Gesellschaft zu erfahren. Das Wissenswachstum erreicht die »Grenzgröße einer alt gewordenen Wissenschaft «, die Kurve flacht nach einer Weile hektischen Oszilierens ab (Solla Price 1974, S. 34 f.).

Freilich gibt es theoretische Grundlagen und Begriffe auch in der Soziologie, die die Hektik dieses Wissensbetriebs unbeschadet überdauern und langfristig die Reflexionskurve markieren, die die Wissenschaft zu ihrem Gegenstand einhält.

Die logistische Kurve ist die des Risikowissens all' dessen, was mit zünftigem Werkzeug zu fassen ist; die Reflexionskurve markiert dagegen das Wissensrisiko, also das, was nie ganz zu fassen ist. Und das ist die Reflexion, ja der Denkzwang, den die Gesellschaft auf die Soziologie ausübt und nicht umgekehrt.

\section{2.}

Seit Tönnies (1887) ist >Gesellschaft $<$ der theoretische Grundbegriff der Soziologie, und es liegt nahe zu fragen, wie er heute verhandelt wird. Tönnies begreift, um ein populäres Mißverständnis auszuräumen, Gesellschaft nicht als Pendant oder Resultat der Gemeinschaft, sondern er spricht von Gesellschaft stets um der Gesellschaft willen - und von Gemeinschaft um der Gemeinschaft willen. Das ist zu betonen z. B. ge- 
gen Max Webers Frage, wann ein Handeln individuell sei und wann sozial, die ja nach wie vor anfällig ist gegen jenes antigesellschaftliche Ressentiment, daß soziales Handeln die Gemeinschaft fördere gegen den gesellschaftlichen Individualismus. Tönnies' Gesellschaftsbegriff ist auch zu betonen z.B. gegen Mannheims Versuch einer Genealogie sozialer »Verhaltungsweisen « vom Finden übers Erfinden zum Planen als ob >Gesellschaft $<$ ein Zustand sei in einer prinzipiell nicht-gesellschaftlich verstandenen Menschheitsgeschichte.

Tönnies dagegen geht der synthetischen Kraft der sozialen Fakten nach, die nicht a priori > positiv < sind, sondern selbst schon reflexiv, also als soziale Fakten immer auch Negationen ihrer empirischen Phänomenologie. Gerade weil das Ganze, wie er sagt, die Teile belebt, sind sie nicht in ihm aufgehoben. »Denn Gesellschaft ist nichts als die abstrakte Vernunft - insofern dieselbe zu wollen und wirken g e $\mathrm{d}$ a $\mathrm{ch} \mathrm{t}$ wird.« (Tönnies 1979, S. 39)

Das heißt z.B.: Gesellschaft ist nicht der einzelne Warentausch, auch nicht die Summe aller Geschäfte, sondern ist der Gedanke, der Begriff des Tauschs, der in ihm steckt - gleichviel, ob die Tauschenden ihn denken oder nicht. Insofern ist dieser reine Grundbegriff eine subjektfreie Denkfigur, die ohne Subjekte >sein<kann, während umgekehrt die Subjekte immer in solche Denkfiguren verstrickt sind, ob sie »wollen und wirken« oder nicht. Mit Adornos Worten: Vom Objekt kann Subjekt weggedacht werden, aber umgekehrt nicht einmal als Idee. (Adorno 1982, S. 184)

Theorie der Gesellschaft ist sozusagen ein genitivus possesivus und kein genitivus objektivus. Die Gesellschaft selbst ist theoretisch und nicht nur Gegenstand einer Theorie. Realabstraktionen wie die Gesellschaft, die Arbeit, das Geld, die Zeit oder die Energie haben durch und durch theoretischen Charakter, den ihre jeweiligen Wirklichkeiten nicht abstreifen können. Ein Geldschein ist nichts ohne den Begriff Geld. Tönnies' »oberster wissenschaftlicher Begriff« der Gesellschaft als abstrakter Vernunft, »welcher nicht mehr den Namẹn von etwas Wirklichem enthält, ist gleich dem Gelde... (wie z.B.) der Begriff Atom oder der Begriff Energie«. (ebda) Angesichts der thermodynamischen Großexperimente, mit denen weltweit die Gesellschaft dabei ist, sich selbst zu zerstören, ist die Analogie aktuell: So, wie es in der Thermodynamik nichts gibt, das nicht dem Begriff der Energie gehorcht, obwohl diese selbst nicht wirklich ist, sondern nur ihre Formen und Zustände (Wärme, Kraft, Geschwindigkeit), so gibt es auch überall, wo Menschen leben, nichts, das nicht einem Begriff von Gesellschaft gehorcht, auch wenn viele Menschen davon nichts wissen wollen.

Je mehr soziologisches Wissen produziert wird, um so schwieriger ist es offenbar, dieses Wissen noch `auf den Begriff zu bringen $<$. Die gesellschaftlich erzeugten Zustände in der Welt üben einen Denkzwang aus, dem die soziologische Theoriebildung offenbar nicht mehr gewachsen ist.

Vielleicht liegt das daran, daß soziologische Theorie es heute zweifach schwerer hat als früher, da - bis Ende der 60er Jahre - der Streit über das, was passiert ist, sie beflügelte, während heute die Einigkeit darüber, was nicht passieren darf, sie lähmt. Die erste Erschwernis ist ein Begriffsverlust: In dem Maß z.B., in dem die entfremdete 
Arbeit und die Fabrik nicht mehr der zentrale Ort gesellschaftlicher Produktivität und politischer Kämpfe um die sog. Lebensqualität sind, erscheinen Begriffe wie Klasse, Arbeit, Entfremdung auch theoretisch als antiquiert. Klasseninteressen gegen Ökologie und Friedensbewegung zu vertreten, ist heute schon fast unmoralisch; und wer an Entfremdung leidet, oder Entfremdung gar für unabdingbar und lebenswichtig hält, der gilt in einer Kultur, die an jeder Ecke mit Selbsterfahrung, Selbstverwirklichung und Selbsthilfe winkt, als hoffnungsloser Fall.

Gravierender ist die zweite Erschwernis: das gesicherte Wissen über die Gefahren und Zerstörungspotentiale. Der gegenwärtigen Zerstörungsproduktion können nicht mehr, wie noch zu Marx' und Tönnies' Zeiten, »zivilisatorische Seiten des Kapitals« abgewonnen werden für eine »höhere Neubildung der gesellschaftlichen Verhältnisse« durch ihre Zerstörung hindurch (vgl. Marx, Das Kapital Bd. III, 48. Kapitel). Heute modernisiert sich der Kapitalismus selbst mit wissenschaftlichen Mitteln, er hat sich seine eigene Kritik einverleibt.

M.a.W.: Nicht mehr darauf hoffen zu können, daß jedes Schlimme sein Gutes habe (der Kapitalismus seine Selbstïberwindung bzw. seinen Zusammenbruch enthalte), ist heute weniger schlimm als die Erkenntnis, daß das Gute selber schlimm ist, nämlich sozialistische Systeme nur noch kapitalistisch saniert werden können.

Der Paradigmenwechsel von der kritischen Theorie des Kapitalismus und der Entfremdung zur Gefahrentheorie der Risikogesellschaft und zur Verteidigung der Lebenswelten gegen die Systeme scheint mithin auch theoretisch kein zu hoher Preis zu sein eingedenk der Erfahrung (es ist Theorie-Erfahrung), daß Gesellschaftstheorie im Banne der Zerstörung diesen nicht bricht, sondern stärkt.

3.

Die gegenwärtigen Gesellschaftstheorien halten zwar in gewisser Weise an der aufklärerischen Tradition der, wie Habermas sie nennt, aälteren Kritischen Theorie < fest, aber sie wollen von einem Gesellschaftsbegriff nichts mehr wissen, der die negative Synthese wäre aus siegreichem Kapitalismus und mißglückter Befreiung. - Negative Synthese als kritische Theorie des siegreichen Kapitalismus im Bewußtsein stets mißratener Befreiung heißt freilich weder nur trotziges Festhalten an Befreiung wider alle Erfahrung des Scheiterns noch einfach Trennung zwischen der Welt der Systeme und den kleinen Freiheiten der Lebenswelten. Negative Synthese wäre vielmehr der Versuch herauszufinden, warum Befreiung als Mittel immer im Sieg-Ziel verschwindet und die Augenblicke der unvermittelten, zwecklosen Freiheit nicht von Dauer sind. Die Befreiung vom Faschismus wurde z.B. in der DDR vor allem als Etappensieg auf dem Weg zum Sozialismus verbucht. Damit wurde Freiheit ein »Problem ... im Lichte des wissenschaftlichen Sozialismus (das Thema der legendären Konferenz in der Deutschen Akademie der Wissenschaften zu Berlin vom 8.-10. März 1956) und nicht umgekehrt der Sozialismus im Lichte der Freiheit problematisiert.

Die Vertilgung der Freiheit durch ihre Sieg-Ziele erzeugt positive Synthesen, wie z.B. den Antisemitismus. Die Juden haben von der bürgerlich-kapitalistischen Freiheit, 
die im 18. Jhdt. die Gettos schliff, wirklich profitiert. Sie verkörperten in den Augen der Subalternen Reichtum und Freiheit, sie waren plötzlich schuld an Armut und Unfreiheit der Anderen. Positive Synthese ist auch das Schicksal, zu denen geschlagen zu werden, zu denen man eigentlich nicht gehören möchte - sowohl >die da unten $<$ als auch >die da oben<. >In $<$ oder >out $<$ zu sein, ist in jedem Fall eine Abfindung damit, Sieger oder Verlierer zu sein.

Angesichts des Alptraums unzerstörbarer Gefahrenherde und Sicherheitszentren (vgl. Seitter 1983, S. 46), mit denen die stärksten und modernsten Gesellschaften sozusagen ihre Befreiung von allen bisherigen historischen Gefahren und Zerstörungen zum Abschluß bringen, ist es verständlich, daß die soziologische Theorie dorthin wandert, wo sie wenigstens noch Teilbegriffe von Gesellschaft, von sich selbst wollender abstrakter Vernunft, erörtern kann. Also in Sphären, die noch nicht vollständig besetzt sind von abrufbarem Wissen. Unter dem Aspekt gesellschaftlichen Wissens wird im folgenden die gefahren- und modernisierungstheoretische Umorientierung der Soziologie angedeutet, mit Hilfe einiger Argumente aus den bekannten Theorie-Unternehmen Ulrich Becks, Jürgen Habermas' und Alain Touraines. Denn diese drei zeichnen sich vor anderen Soziologen dadurch aus, daß sie an einem Gesellschaftsbegriff festhalten, auch wenn sie mit ihm die Soziologie verlassen müssen. Bei Habermas ist der Gesellschaftsbegriff in die politische Publizistik gewandert und lebt wieder auf in seinen großen politischen Essays. Bei Beck ist er weniger in der Risiko-Diagnostik als im moralischen Diskurs zu finden, und Touraine, der Klassentheorie kritisch verbunden, versucht Gesellschaft als das Interesse sozialer Akteure zu begreifen, freilich weit entfernt vom Gleichgewicht modischer Handlungstheorien.

Bei Ulrich Beck heißt es:

»In Gefährdungslagen sind also - anders als in Klassenlagen - Lebenslagen und Wissensproduktion direkt ineinander verschoben und verschränkt. Daraus folgt: Die ... Theorie der Risikogesellschft ist in ihrem Kern Wissenssoziologie, eben ... Soziologie a 1 le r Wissensbeimengungen, Wissensamalgame und Wissensakteure in ihrem konfliktvollen Ineinander und Gegeneinander ... und ihren Unmöglichkeiten, das Wissen, das sie beanspruchen, zu wissen.« (Beck 1986, S. 72)

»...Die moderne Gesellschaft hat kein Steuerungszentrum« (S. 368) und »wo alles verfuggbar geworden ist, ist das Zeitalter der Ausrede vorbei « (S. 372)

Nicht das Bewußtsein, aber das Wissen bestimmt das Sein, und auf die Klassenlage kann sich auch niemand mehr hinausreden.

Auch Touraine definiert Modernisierung als »die wachsende Fähigkeit der Gesellschaft, auf sich selbst einzu wirken« (Touraine 1982, S. 288 und 1986, S. 18), womit, ganz im Gegensatz zur Systemtheorie, eine »Dissoziation von Akteur und sozialer Situation $\ll, z . B$. seiner Klassenlage, einhergehe und eine $»$ Demontage des Gesellschaftsbegriffs « bisheriger Klassen- und Struktursoziologie erforderlich werde. Um des »sozialen Denkens « willen müsse zwischen »Erforschung des Akteurs und Erforschung der Gesellschaft« getrennt werden, denn »das Interesse des Individuums und das Interesse der Gesellschaft fallen nirgends mehr zusammen (1986, S. 33 f.). Das ist freilich ein anderer Diskurs als bei Beck, der die individuellen und die Klasseninteressen mit den Überlebensinteressen in der globalen Risikogesellschaft zusammenfallen sieht. >Raus aus der Klasse und hinein ins gemeinsame Boot< ist nicht 
Touraines Parole. Er diagnostiziert vielmehr verschärfte Konflikte zwischen Klassenlage und Wissen.

»Information und Kommunikation erscheinen so gesehen nicht als technisch komplementäre, sondern als sozial entgegengesetzte Begriffe. Wer von der Informationsgesellschaft spricht, spricht vor allem von der Konzentration und Macht der Informationsmittel, der-produktion und -verbreitung. Der Begriff der Kommunikation hingegen stellt die Versuche der sozialen Akteure, Mitteilungen auszutauschen und zu ... verändem, in den Vordergrund. Die Welt der Kommunikation ist dezentralisiert, vielfältig und veränderlich, die der Information hingegen standardisiert und auf die Steigerung des Nachrichtenflusses und der Übermittlungsgeschwindigkeit ausgerichtet.« Thre Akteure seien »die neuen sozialen Bewegungen ... in Opposition gegen die Informationsgesellschaft.« (Touraine 1986, S. 25)

Und das sind bei Touraine keine technikfeindlichen, alternativen Fluchthorden und auch keine besonders betroffenen Risikogruppen, sondern eher moderne, mobile und qualifizierte Bescheidwisser und Durchblicker, deren »Soziologie des Handelns ... mit der Soziologie der Gesellschaft in der Krise gebrochen hat (S. 35). Deren Handeln ist auch eher ein Nicht-Handeln. In Touraines Soziologie steht nicht »ein durch seine soziale Rolle definierter Akteur wie der Arbeiter oder der Staatsbürger (S. 36) im Mittelpunkt, sondern ein politisches Subjekt, das zu herkömmlichen Organisationen, Gemeinsamkeiten und Werten auf Distanz geht und "sichtbarer wird in dem, was es unterläßt als in dem, was es tut...«. (S. 35).

Der Witz dieser Soziologie des Handelns ist, daß sie nicht die Konflikte unterschiedlicher Kräfte um die Verwirklichung einer gemeinsamen Sache erörtert, wie z.B. die Kämpfe zwischen Arbeiterbewegung und Kapital um den Fortschritt der Industriegesellschaft. Vielmehr versucht sie, aus der »kulturellen Gemeinschaft« z.B. der Atomtechnokraten und der AKW-Gegner, sozusagen aus der Wissensnotgemeinschaft der Betreiber und der Gegner, einen permanent strittigen, in sich unvereinbaren Begriff der postindustriellen Gesellschaft zu reflektieren. Beide partizipieren an der »großen Mutation« zur programmierten Wissensgesellschaft, aber die einen setzen auf den Ausbau der technologischen Spitzenindustrien und auf Globalsteuerung, die anderen auf das wissenssoziologische »Bündnis von Modernisierung und demokratischem Protest « (vgl. Touraine 1982, S. 287 f.). In der Tat scheinen die »wissenschaftlichen Erkenntnispraktiken « (Beck, S. 284) so unvereinbar, daß die »neuen Konflikte«, von denen Touraine und Habermas sprechen (Touraine 1982, S. 310 ; Habermas 1981, Bd. 2, S. 581), zum Glück nicht einfach entschieden werden können, sondern der postindustriellen Gesellschaft ihre Dynamik und der Soziologie ihre sub- oder nebentheoretische Permanenz verleihen.

$>$ Kultureller Wandel wird nur durch soziale, politische Aktion auch zu sozialem Wandel.

»Eine Subkultur z.B. kann fortgesetzt neue Ideen erzeugen, und dennoch keine signifikanten Veränderungen in der sozialen Struktur bewirken. ... das Glied, das Kultur und soziale Struktur verbindet, ist die politische Aktion.« (Sebald 1988, S. 120)

Ulrich Beck bemerkt ironisch, daß selbst fundierte und massenhafte Kritik an den »risikointensiven Großtechnologien«, z.B. die Hearings in Wackersdorf, wie »ein Nachruf « wirke. »Man kann zum Fortschritt zwar nein sagen, aber das åndert nichts an seinem Vollzug ... jenseits von Zustimmung und Ablehnung (Beck, S. 329). Der 
kulturelle Dissens der Protestierenden und die risikokritische Wissenspraxis, die z.B. die sog. Nebenfolgen zur Hauptsache der Konflikte machen, erlauben es jedoch, diesen Satz auch umzudrehen: Ja zum Fortschritt in Theorie und Wissenschaft, damit man nicht erst aus Schaden klug wird. Bei manchen Sachen ist bekanntlich jeder Fehlversuch einer zuviel.

Freilich wächst mit jedem Wissenszuwachs auch das Unvorhersehbare und die Verwirklichung jeden Wissens vertilgt weitaus mehr Mittel - nicht nur Geld - als im Zweck schließlich erscheinen.

4.

Die »wissenschaftlichen Erkenntnispraktiken «, über die es in der Gesellschaft Streit gibt, haben zunächst ein gemeinsames Prinzip: Das Denkbare ist auch machbar; jedenfalls wird darüber nachgedacht, ob es auch zu machen ist. Der Streit beginnt mit der Frage, ob auch alles gemacht werden darf - und wird soziologisch, wenn es darauf keine eindeutige Antwort mehr gibt, sondern Begründungskonflikte ausgetragen werden z.B. zwischen ökonomischen und moralischen Interessen. Diese wiederum ändern sich manchmal rasch. Drei Beispiele:

1. Um Erfolge bei Organverpflanzungen und Strahlenkrebstherapien erzielen zu können, müssen massenhaft Organe und Krebsfälle geliefert bzw. produziert werden. Beides, die unvermeidlichen Übel des Zulieferbetriebs (Unfallopfer, Leben im Atomzeitalter) und die Erfolge sind heute in gewissen Grenzen akzeptiert. Bei Transplantationen ist bislang noch der Kopf tabu, und beim Strahlenkrebs die nächste große Atommeiler-Katastrophe in Greifswald, Biblis oder Gravelines.

In manchen Elendszentren der südlichen Erdhälfte ist das Kapital aber nicht mehr an der Arbeitskraft und der Gesundheit der Menschen interessiert, sondern an ihren Letztelementen, d.h. an ihrem Blutplasma und ihren Organen. Nicht mehr Soldaten und industrielle Reservearmeen sind gefragt, sondern Ersatzteilpopulationen. Auch auf diese Weise modernisiert sich der Kapitalismus wissenschaftlich, betreibt systemische Selbsterhaltung um den Preis humaner Orientierung. --Die Frage ist nur, wann dies zum Paradigmenwechsel auch in der Soziologie wird, z.B. als Wertewandel in den neotropischen Klimaflucht-Gesellschaften des 21. Jhdts. Der sog. Diskurs der Lebenswelten und Lebensstile hat sein Thema zu Zeit noch gar nicht gefunden.

2. Die meisten sind heute dafür, daß - koste es was es wolle - an Medikamenten und Therapien geforscht wird, die das Immunsystem von AIDS-Kranken wieder regenerieren. Der gesellschaftliche Fortschritt auf die Lustseuchen-Dämonologien früherer Epochen ist, daß auf diesem Gebiet Strafjustiz und Kirchen weitgehend ausgespielt haben; jetzt muß Wissenschaft her. Der gesellschaftliche Streit beginnt wahrscheinlich dann, wenn die Immunologie Genmanipulationen vorschlägt. Jede >Lösung< des Problems wäre freilich auch hier positve Synthese: nämlich die gesellschaftliche und individuelle Versöhnung des Unglücks mit seiner Bewältigung. Ein Gesellschaftsbegriff wäre erst noch zu denken, der die negative Synthese wäre zwischen kapitalistisch-wissenschaftlichem Siegeszug und wiederum mißglückter sexueller Befrei- 
ung. Negativ und d.h. theoretisch angemessen wäre es nämlich, die Bewältigungspraktiken selbst als Unglück zu begreifen. Wer AIDS hat, mit Ersatzteilen leben muß oder Verstrahlung ausgesetzt war, zieht seine Lebenskraft, inklusive der Hoffnung auf Heilung, aus dem Unglück, das ihm widerfahren ist, aber nicht aus den stets prekären Heilerfolgen. Man kann nicht ein Übel mit einem anderen Übel bekämpfen, sondern das erste hält das zweite aus.

3. Von den »positiven Seiten « der neuen Technologien haben die Wortführer der Studentenproteste 1968 wahre Wunder erwartet. Mit Automation und Mikrochips werde die Arbeitszeit auf ein Minimum reduziert; die Computertechnologie mache die Bürokratie und die Justiz überflüssig. »Intelligente Industriezweige « in den richtigen Händen: »Wenn es die Computer nicht gäbe, müßten sie förmlich erfunden werden für die Räteverfassung « (Kursbuch 14, 1968, S. 168). Die Gegner der atomaren Aufrüstung forderten die friedliche Nutzung der Kernenergie. Und das, was seit Solidarnosc und Gorbatschow im ehemaligen Ostblock passiert und Mitte der 60er Jahre von Analytikern im State Department und MIT schon recht genau prognostiziert wurde, wurde $1968 \mathrm{im} \gg$ Kursbuch noch ganz klar zur $\gg$ Zukunft der Konterrevolution« gezählt (vgl. Kursbuch 14, S. 20 f.). Irrtümer über Irrtümer -- aber davon lebt Theorie. Daß die jetzt abgetretenen ML-Greisenregimes die Welt nicht mehr verstehen, liegt auch am Theorieverlust des sog. wissenschaftlichen Sozialismus.

Soziologische Wissensproduktion müßte mithin reflektieren, daß mit jeder Zielverwirklichung eine Entwirklichung der Mittel einhergeht, daß deren »Wirklichkeitsvorsprung « in dem Maß schwindet, wie sie ihrem Soll-Zustand zugeführt werden (vgl. Seitter 1985, S. 141). Der sog. »real existierende Sozialismus « der DDR wurde immer unwirklicher, je mehr davon existierte. Je mehr Sicherheit produziert wird mit Waffensystemen und Sicherheitsgesetzen, desto unsicherer das Ganze; je mehr Verrechtlichung des sozialen Lebens zum abstrakten Schutz der Grundrechte und Freiheiten, desto weniger können die Bürger sie genießen (vgl. Habermas 1981 Bd. 2, S. 540 f.). Und je mehr Bürokratie und Justiz mit elektronischer Datenverarbeitung ausgestattet werden, um so dringender sind unabhängige Richter und lebendige, unberechenbare Zeugen nötig.

5.

Soziologische Theorie, die den Namen verdient, sollte sich an einem snegentropischen< Gesellschaftsbegriff orientieren. D.h.: Der »Wirklichkeitsvorsprung « der gefährlichen, strittigen, ungerechten und aufregenden Zustände vor einem »Sollzustand « ubiquitären Datenwissens und technischer wie gesetzlicher Totsicherheiten ist zu vergrößern. Das wäre Gesellschaftstheorie, die weder kapituliert vor den selbstproduzierten Datenbergen und Zwangsvorstellungen des Wissenmüssens, noch auf postmoderne Science Fiction sich kapriziert.

Auch Habermas umkurvt, was den Ort der Theoriebildung »kommunikativen Handelns « angeht, dieses paradoxe gefahrentheoretische Axiom: Die Verhinderung von Katastrophen hängt ab vom Katastrophenwissen, dessen Voraussetzung viele Kata- 
strophen sind. M.a.W.: Weil viel kaputt geht, geht immer mehr nicht kaputt, wie z.B. im Autoverkehr. 1970-72 war Autofahren in der Bundesrepublik am gefährlichsten. Seither nimmt die Verkehrsdichte zu und die Menge der Unfälle mit Personenschäden ab (vgl. FR, 24.2.90).

Das Problem ist nur, daß der Autoverkehr heute zu einer unzerstörbaren Gefahrenquelle neuer Qualität geworden ist.

Die »Zerstörungsprozesse, die den Weg der kapitalistischen Modernisierung gesäumt haben« (Habermas 1985, S. 189), machen gegenwärtig diesen qualitativen Sprung: heute werden zunehmend »nicht-regenerierbare Bestände« der Lebenswelt den »systemischen Imperativen « der Rationalisierung, Programmierbarkeit und Sicherheit geopfert. (Vgl. Habermas 1981 Bd. 2, S. 550)

Ähnlich wie Touraine formuliert Habermas den Unterschied zwischen Information und Kommunikation als soziale Konfliktlinie:

»Die Frontlinie zwischen Lebenswelt und System bekommt damit eine ganz neue Aktualität. Heute dringen die über die Medien Geld und Macht vermittelten Imperative von Wirtschaft und Verwaltung in Bereiche ein, die irgendwie kaputt gehen, wenn man sie vom verständigungsorientierten Handeln abkoppelt und auf solche mediengesteuerten Interaktionen umstellt.« (Habermas 1985, S. 189)

Diese Zerstörung der Lebenswelt durchs System hat wissenstheoretisch gesehen zwar den Vorteil, Wissen produziert zu haben, aber das derart Gewußte ist jetzt leblos. Zwar ist der Mensch mehr als die Summe seiner Daten, aber die zählen als Information. Gegen diese Wegrationalisierung des Lebens mobilisiert die Lebenswelt jedoch ihre eigene »kommunikative Rationalität«als selbstreflexive Fähigkeit, bisherige Gewißheiten fahren zu lassen.

»Die Lebenswelt ist das merkwürdige Ding, das vor unseren Augen zerfällt und verschwindet, sobald wir sie stückweise v o r u n s bringen wollen. Die Lebenswelt funktioniert als Ressource für das, was in explizite Äußerungen eingeht; aber in dem Augenblick, wo dieses Hintergrundwissen ... zu einem expliziten Wissen und damit kritisierbar wird, verliert es gerade den Gewißheitscharakter...«. (Habermas 1985, S. 186)

Kommunikative, moderne Lebenswelt mithin, die in der Lage ist, reflexiv sich selbst zu thematisieren, fällt nicht dem System zum Opfer, sondern öffnet sich dem »erweiterten Horizont « des Ungewissen (ebda). Nur dieses Herausfallen aus sich selbst bewahrt sie davor, lebensphilosophisch oder postmodern hocken zu bleiben in den Lücken des Systems. Und das ist nicht, analog etwa zu Luhmanns System-UmweltDifferenz-Theorie, eine Theorie zweier komplementärer oder divergenter Welten, sondern Theorie als Reflexionsform des Verhältnisses der denkenden, handelnden und leidenschaftlichen Subjekte zur Welt, zur Gesellschaft, zur menschlichen Natur (vgl. Habermas 1985, S. 185).

Habermas' Theorie vernünftiger Kommunikation, Touraine's Theorie der sozialen Akteure und auch Becks »Risikogesellschaft « sind, wie gesagt, gerade dort Theorien der Gesellschaft, wo sie aus dem Ganzen in Teile wandern, die genau durch diese Theoriebeatmung nicht mehr im Ganzen aufgehoben sind. Habermas' politische Publizistik z.B. im sog. Historikerstreit und seine wache Kritik konservativer Grundrechte-Beschädigungen konfrontieren unsere real existierende Demokratie mit ihrem eigenen Anspruch. Touraines Analyse z.B. der französischen Antinuklear-Proteste 
verunsichert nicht nur den französischen Sicherheits-Konsens, sondern auch den selbstgefälligen linken Habitus des Dagegenseins. Und Becks moralisch-diagnostischer Diskurs wird immer dort theoretisch, wo er umschwenkt von der wissensbeladenen Kritik der »Gefährdungslagen« zur `gefährlichen〈, weil theoretischen folgenreichen Kritik liebgewordener Gewißheiten z.B. über die moderne Kleinfamilie oder monokausale Gefahrenbeweise.

An diesen Orten enthalten die soziologischen Unternehmen Spuren, Schwundspuren gewissermaßen, dessen, was einst die Kritische Theorie auszeichnete: nämlich die bestimmte Negation des Gesellschaftsbetriebs bei gleichzeitiger Sympathie mit dem sozialen Leben, das ihn aufrechterhält. In dieser Sympathie, nicht im empirischen Wissen über den Betrieb, steckt die Hoffnung auf soziale Konkretion des Ungewissen und Unwissbaren, nämlich der Selbstrettung der Gesellschaft.

Habermas versucht freilich, die Kategorie des »verständigungsorientierten Handelns « mit Hilfe der Marxschen Realabstraktion der Kategorie >Arbeit $<$, der »Arbeit überhaupt «, zum obersten theoretischen Begriff zu machen, der auch noch den verständigungsfeindlichsten Problemen und Konflikten innewohnt.

Erkennende und kommunizierende Vernunft in ihrer handelnden, denkenden, redenden und leidenden Beziehungsform ist, so hofft Habermas, heute die Form der Theorie, die »der ratio essendi ihrer Gegenstände gewachsen ist (Habermas 1981, Bd. 2, S. 590). - Schwächer als diese, aber mit bezwingender und erzwungener Reflexionskraft. Diesen Denkzwang übt das Ganze aus, dem es egal ist, ob es mit dem Hegelschen Totalitätsbegriff traktiert wird oder ob System- und Handlungstheorie mit getrennten Bestecken sich an ihm zu schaffen machen.

Die Systemtheorie leugnet diesen Denkzwang; sie setzt auf die Entlastungseffekte der Rationalisierung. Die Informatik des Systems nimmt den Leuten in der Lebenswelt nicht nur die Entscheidungen ab, so daß sie gedankenlos kommunizieren können. Sondern sie löst auch die, wie die Juristen sagen, >natürliche Handlungseinheit< aus Intention-Mittel-Zweck-Kosten auf zugunsten scheinrealer empirischer Handlungsschnipsel bzw. »Letztelemente «, die je für sich »anschlußfähig «sein sollen. Deshalb braucht Luhmann auch keinen Handlungsbegriff, sondern eine Schere, ein SystemUmwelt-Differenzier-Werkzeug.

Beck, Touraine und Habermas dagegen betonen, daß die Rationalisierungsgewinne des Systems »die kommunikative Infrastruktur der Lebenswelt «überlasten (Habermas 1981, Bd. 2, S. 554). Das Leben wird durch Automatisierung und Rationalisierung, durch jederzeit abrufbares Wissen, nicht leichter, sondern reflektierte Beziehungsformen in der Lebenswelt und mit dem System werden immer schwieriger.

6.

Hier beginnt das Dilemma der Theorie.

In der gegenwärtigen »Gefahrenlage« einer denkbaren »Selbstauslöschung der Menschheit« verlangt z.B. Johannes Berger von der Soziologie die »systematische Erforschung « all dessen, was heute Modernisierung heißt. Um beispielsweise über das zu- 
künftige »Verhältnis von Arbeit und Leben« etwas sagen zu können, »bedürfte es allererst eines gesicherten Wissens darïber, worin denn der Grundvorgang der Modernisierung besteht $\ll$. (Berger 1986, S. 7 f.)

Systematisch erforscht werden kann freilich nur das, was passiert ist und das, was in der Macht der Forscher steht: was sie tun und lassen, ihre eigenen sozialen Abenteuer; wie z.B. Touraines und seiner Mitarbeiter Beteiligung an der französischen AntiatomBewegung. Nun sind die Prozesse der Modernisierung solcher Forschung immer um einige Schritte voraus - und da, wo Soziologen oder andere Leute qua Beteiligung in die Modernisierung direkt verstrickt sind, ist ihr Wissen darüber notwendig unsicher und sind Aussagen über zukünftige Verhältnisse ungewiß.

Was man sagen kann, ist nur, daß der »Grundvorgang der Modernisierung « in der Dynamik des wissenschaftlichen Kapitalismus besteht, alles in der Welt durchzukapitalisieren und die Zukunft schon mitzuvertilgen, bevor sie eine Chance hat. Mithin bedürfte es »allererst« störender Eingriffe in die Sicherheit, mit der der Kapitalismus nicht nur Kapital und Politik, sondern auch die Wissenschaften benutzt, um sich auf Kosten aller Lebens- und Arbeitsverhältnisse zu modernisieren.

Zu den »nicht regenerierbaren« Beständen des sozialen Lebens gehören jedoch nicht nur die sog. Umwelt und die kommunikative Kultur, sondern auch die humane Orientierung der Wissensproduktion und Gefahrenforschung.

Und die geht offenbar verloren in den Allmachtsphantasien der neuen geopolitischen Gefahrenforschung und Wissenspolitik:

$»$ Das Ozon in der Atmosphäre (habe) ... schneller abgenommen als Modellrechnungen vorausgesagt haben. Deshalb müsse die Forschung insbesondere auf der Nordhalbkugel verstärkt werden, sagt Riesenhuber ... in Bonn. Während die Daten des Ozon-Abbaus auf der Sïdhalbkugel ausreichend bekannt seien, wisse man für die Gebiete nördlich des Äquators nur, daß dort das Ozon »in den letzten zehn Jahren um rund drei Prozent abgenommen hat«. Pflanzen würden möglicherweise mehr noch als Menschen unter der verstärkten Ultraviolettstrahlung leiden, sagte der Minister. Das könne zu einem Rückgang der Ernteerträge führen... Die notwendigen Forschungsdaten für die nördliche Halbkugel sollen vom Forschungsschiff $>$ Polarstern<, Klima-Bailons, einem $15 \mathrm{~km}$ hoch fliegenden Flugzeug und Satelliten geliefert werden. « (aus FR vom 19.7.1988, S. 4)

Hier wird, mit Horkheimers Wort, versucht, das Schlechte (Ozonloch) durch das Gute (Wissenschaft) zu definieren statt abzuschaffen. »Notwendige Forschungsdaten« wären dagegen solche, die z.B. einer kompromißlosen Produktions- und Emissionsverbotspolitik abgewonnen würden; und zwar mit dem Wissensrisiko, das auch ein politisches und ökonomisches Risiko ist, vorher nicht sagen zu können, ob solche Verbote auch den gewünschten Effekt haben. Die Forschungsdaten, die auf den Expeditionen des Bundesministeriums gewonnen werden, sind dagegen gewiß solche, die jede industriell unerwünschte Wissenspolitik verzögern und die bereits bekannten irreversiblen Schäden etablieren. Die Forschungspolitik des derzeitigen Ministers begünstigt also nicht kritisches Nichtwissen aus Versuchen, die Gefahrenproduktion einzuschränken, sondern sicheren Gefahrenzuwachs durch Wissensproduktion.

Und das ist wissentliche Produktion des Nichtwissens. Das nämlich, was man wissen müßte, ob z.B. durch eine ökologische Umorientierung der Industrie, der Forschung, der Konsumgewohnheiten die globalen Gefahren wirklich gebremst würden, das 
wird durch die >systemischen Imperative < der Gefahrenforschung und -produktion selbst verhindert.

Zwischen humaner Orientierung, zu der auch ein Leitwert, ein Begriff intakter Atmosphäre gehört, und den Vergiftungs- und Strahlengrenzwerten, die als tolerabel verordnet werden, herrscht nicht mehr die nötige Diskrepanz. Die verschwindet bereits in dem Maß, wie die Grenzwerte die Leitwerte verdrängen, wie >Akzeptanz und $>$ Resistenz $<$ sprachlich und sozial sich einbürgern anstelle von Konflikt und Widerstand, von Empfindlichkeit und Konsenskriterien. Die derzeit begünstigte Wissensproduktion über die großen Gefahren ist so gefährlich wie theorielos und so kapitalintensiv wie zukunftstilgend. Es herrscht, mit einem Wort, Information ohne Kommunikation. Gefragt im Blick auf zukünftige Verhältnisse ist also nicht »gesichertes Wissen « darüber, wann das Loch im Himmel so groß ist, daß wir das Tageslicht besser meiden, sondern nötig ist der materielle und theoretische Konflikt zwischen denen, die die Zukunft so belasten und besetzen, daß es in ihr keine Fragen, nur noch Antworten gibt, und denen, die lieber die Probleme vererben als die Lösungen.

Dabei braucht die hier erwähnte Soziologie ihr Licht nicht unter den Scheffel zu stellen: Touraine, Habermas, Beck, auch Bergers theoretisches Engagement für die Grünen, liefern dem Gesellschaftsbetrieb nicht einfach Wissen, sondern versuchen, ihm theoretisch standzuhalten unter antitheoretischen Bedingungen.

Es wäre gefährlich und antitheoretisch, besonders für gefahrentheoretisch fundierte Humanwissenschften, die sog. Grenzwerte und >zulässigen< Höchstbelastungen als dauerhafte Leitwerte hinzunehmen, oder den Tieren und Pflanzen vorzuwerfen, mit der Resistenzfähigkeit der Menschen nicht mithalten zu können. Solch fatale Gewöhnungsprozesse machen, wenn sie nicht aufpaßt, auch vor der Soziologie nicht halt. Gerade das, was unmerklich akzeptiert wird und wogegen soziales Denken und Empfinden resistent werden, bereitet den Boden für inhumanen Wertewandel und für $>\mathrm{Mo}$ dernisierungsschäden kompensierende< Paradigmenwechsel. Die Wahl der Schlüsselbegriffe und der Wissenswünsche steuert bekanntlich »die Analyse selbst und ihre Resultate in bestimmter Richtung « (Adorno 1986, S. 670). Alle wesentlichen Daten produzieren zu können über die Entwicklung des Ozonlochs - oder über die Modernisierungsdynamik der reichen Industriegesellschaften, ist zwar ein verständlicher Wissenswunsch. Die Wissenschaft wäre dann endlich einmal stärker als ihr Gegenstand, sie hätte alles im Griff. Dieser Wunsch, sozusagen noch zu Lebzeiten möglichst viel Zukunft zu erleben, ist freilich fatal. Er ist das Eingeständnis, den Tod wissen zu wollen. 


\section{Literatur}

Theodor W. Adomo (1982): Negative Dialektik, Frankfurt/M.

ders. (1986): Gesammelte Schriften, Bd. 20.2, Frankfurt/M.

Roland Barthes (1980): Leçonilektion, Frankfurt/M. *

Ulrich Beck (1986): Risikogesellschaft, Frankfurt/M.

Johannes Berger (1986): Die Modeme - Kontinuitäten und Zäsuren; Soziale Welt Sonderband 4; Göttingen

Elvio Fachinelli (1981): Der stehende Pfeil. Drei Versuche, die Zeit aufzuheben. Berlin

Jürgen Habermas (1981): Theorie des konmunikativen Handelns, 2 Bände. Frankfurt/M.

ders. (1985): Die neue Unübersichtlichkeit, Frankfurt/M.

Kursbuch 14 hg. v. H.M. Enzensberger (1968): Kritik der Zukunft, Frankfurt

Niklas Luhmann (1984): Soziale Systeme. Grundriß einer allgemeinen Theorie. Frankfurt/M.

Karl Marx (o.J.): Das Kapital, 3. Band, MEW Bd. 25, Berlin (DDR)

Hermann Pfütze (1987): Antike Logik - Moderne Hektik. Zur Tradition subjektloser Denkfiguren in der Soziologie. In: Lars Clausen, Carsten Schlüter (Hg.), loo Jahre Gemeinschaft und Gesellschaft. Im Erscheinen

Herbert Schnädelbach (1989): Die Aktualität der >Dialektik der Aufklärungく. In: H. Kunneman, H. de Vries (Hg.), Die Aktualität der ,Dialektik der Aufklärungく. Frankfurt/M.

Hans Sebald (1988): New-Age-Spiritualität. In: Kursbuch 93

Walter Seitter (1983): Der große Durchblick. Berlin

ders. (1985): Menschenfassungen. Studien zur Erkenntnispolitikwissenschaft. München

Derek J. de Solla Price (1974): Little Science, Big Science. Frankfurt

Ferdinand Tönnies (1979): Gemeinschaft und Gesellschaft. Neudruck Wiss. Buchgesellschaft Darmstadt

Alain Touraine u.a. (1982): Die antinukleare Prophetie. Zukunftsentwürfe einer sozialen Bewegung. Frankfurt/M.

ders. (1986): Krise und Wandel des sozialen Denkens. In: Soziale Welt Sonderband 4 Göttingen 